\title{
Artificial Intelligence for Healthcare in Africa
}

\author{
Ayomide Owoyemi $^{1 *}$, Joshua Owoyemi ${ }^{2}$, Adenekan Osiyemi $^{3}$ and Andy Boyd ${ }^{1}$ \\ ${ }^{1}$ Department of Biomedical and Health Informatics, University of Illinois at Chicago, Chicago, IL, United States, ${ }^{2}$ ZMP Inc., \\ Tokyo, Japan, ${ }^{3}$ Department of Family Medicine, University College Hospital, Ibadan, Nigeria
}

Keywords: Africa, artificial intelligence, digital health, machine learning, sustainable development goals, data

\section{INTRODUCTION}

Digital technology will play a significant role in achieving sustainable human development worldwide. In 2015, United Nations Member States set 17 goals, the Sustainable Development Goals (SDGs), to provide a road map for the achievement of Earth's peace and human prosperity by 2030. SDG 3, as one of the goals which is aimed at ensuring healthy lives and promoting well-being for all at all ages, will greatly benefit from the implementation of digital technology. With over a billion people, Africa can be better positioned to surmount its health challenges-especially regarding maternal and child health and infectious and non-communicable diseases-using digital technology including artificial intelligence (AI).

AI is defined as the automation of activities associated with human thinking such as decision-making, problem-solving, and learning (1).

AI was first used in medicine in the 1970s when medical expert systems-based on Bayesian

OPEN ACCESS

Edited by:

Florian B. Pokorny,

Medical University of Graz, Austria

Reviewed by:

Vignesh Subbian,

University of Arizona, United States

Karmen S. Williams,

Indiana University, United States

*Correspondence:

Ayomide Owoyem

aowoye3@uic.edu

Specialty section:

This article was submitted to

Health Informatics,

a section of the journal

Frontiers in Digital Health

Received: 23 February 2020

Accepted: 08 May 2020

Published: 07 July 2020

Citation:

Owoyemi A, Owoyemi J, Osiyemi A and Boyd A (2020) Artificial

Intelligence for Healthcare in Africa.

Front. Digit. Health 2:6.

doi: 10.3389/fdgth.2020.00006 statistics and decision theory-diagnosed and recommended treatments for glaucoma and infectious disease (2). Progress in Bayesian networks, artificial neural networks, and hybrid intelligent systems in the late 1990s has scaled up bioinformatics research, thereby expanding uptake of medical artificial intelligence (MAI) (3). Global investment in MAI is projected to hit about $\$ 6.6$ billion by 2021 as it is anticipated that AI implementations in healthcare can help save $\$ 150$ billion in costs by 2026 (4).

At present, a more meaningful application of MAI occurs in developed nations compared with what is obtained in Africa. The United Nations in two different forums has signaled a need to change this narrative by bringing stakeholders together to discuss how AI can be used to deliver critical public services and help in the journey toward achieving the SDGs (5). In this paper, we briefly highlight contemporary MAI use in Africa, along with its opportunities, challenges, and likely prospects.

\section{CONTEMPORARY ARTIFICIAL INTELLIGENCE FOR HEALTHCARE IN AFRICA}

Historically, MAI was piloted in Africa in the mid-1980s. MAI deployed in Kenya improved health worker-patient interaction quality with evidence of increased number of symptoms elicited (6). To improve the detection of common and potentially blinding eye disorders, MAI (successfully implemented in the US) was piloted in Egypt some time in 1986 (7). Similarly, in the Gambia, a probabilistic decision-making system assisted rural health workers to identify life-threatening conditions in outpatient clinics. The MAI performed tolerably well in detecting $88 \%$ of cases. Computerized Aid To Treat (CATT) was also used in drug prescriptions in South Africa by nurses based on a cost-and-effectiveness algorithm $(8,9)$.

In recent times, the healthcare application of AI in Africa has only seen a few pilots and test cases. For example, in South Africa, a multinomial logistic classifier-based system is being applied 
to human resource planning, especially to predict how long health workers might stay in public service (10). A partnership comprising researchers and a social enterprise has been developing an AI planning application for optimizing the scheduling of community health workers (CHWs) in communities in Africa (11).

In Nigeria, Ubenwa is a start-up that is using signal processing and machine learning to improve the diagnosis of birth asphyxia in low-resource settings (12). Bellemo et al. (13) conducted a study in using AI to diagnose diabetic retinopathy in Zambia which showed significant and promising results when compared with human assessments. It showed clinically acceptable performance in detecting referable diabetic retinopathy (13). The Delft Institute's CAD4TB software has been employed in pilot studies examining the use of a computer-aided diagnosis of pulmonary tuberculosis from chest radiographs in Tanzania and Zambia. The results are exciting as the performance of CAD4TB compared well with that of human experts $(14,15)$.

Pharmaceutical industries have also benefited from the use of AI in Nigeria. A group of five high school girls developed an app based on an MIT open-source software to identify fake drugs in Nigeria. Their stellar idea received recognition in 2018 upon winning a Silicon Valley contest. Earlier in 2019, Adebayo Alonge, a Nigerian trained pharmacist won the grand prize of Hello Tomorrow Global Challenge 2019 for his AI-hyperspectral platform for authenticating drugs $(16,17)$.

\section{DISCUSSION}

\section{Issues Around Implementing Artificial Intelligence for Healthcare in Africa Data Availability and Quality}

Machine learning and precision medicine applications require a training dataset for which the outcome variable (e.g., onset of disease) is known. An important challenge in applying AI for health and medicine is the lack of large clinical datasets for training AI models. This is especially true for datasets with labels, which require doctors/medical expert annotation and therefore are costly and time-consuming to collect (18). With the low level of digitization and electronic medical record use across Africa, there is a paucity of locally generated useful data that are important for building AI systems (19).

The concept of algorithmic bias-which implies that AI is as good as the data on which it is trained on-is an important factor in the development of AI products and applications. This factor, though global and not just an African problem, has a more pronounced effect when AI applications are introduced to the African setting (20). Because most AI applications are being developed outside Africa, most datasets available are from people who differ from Africans physiologically. While this might not be a big issue as seen in the study done on diabetic retinopathy, it might affect the sensitivity adversely in other cases (13). AI systems could also contain algorithms that stem from the prejudices and specific beliefs of the creators of the AI systems. This introduces the possibility of unintended bias which creates potential discriminations and wrong outcomes if applied to others in low-resource settings without their developmental input and data (21-23).

\section{Legal and Policy Issues}

Across the world, a lot of countries are just developing a governance policy or legal framework for the implementation of AI in different sectors including healthcare $(24,25)$. Some countries in Africa do not have a national digital health policy or strategy which will guide the implementation and monitoring of digital health strategies. African stakeholders also believe that low government engagement at the policy level has stymied adoption, and a stronger engagement will encourage early adoption of AI and other digital tools (26).

In Africa and around the world, there are no enacted laws guiding who takes responsibility for adverse outcomes that might result from the usage of AI in healthcare which is highly likely considering how and where AI might be applied in healthcare. The most probable resolutions might involve the application of existing laws, but some areas and scenarios are not anticipated or covered by the existing laws. This will have legal implications for users and patients in these African countries (22).

\section{Costs of Artificial Intelligence Application and Adoption}

The cost of assembling an AI/ML-based solution in healthcare is difficult to ascertain in developed countries and could range from as low as $\$ 6,000$ for simple chatbots to as high as millions of dollars (27). Costs associated with the application or adoption of $\mathrm{AI}$ are as follows:

1. Data acquisition and preparation costs: This involves data collection proper and cleaning up and annotation of data. The quality of an AI application is mostly dependent on the quality of data on which it is built. Therefore, this stage is usually critical and time-consuming and can also be very costly depending on the granularity of the information required to be extracted from the collected data. COCO, a large-scale object detection, segmentation, and caption dataset, contains more than 200,000 labeled images containing semantic and instance information of the contents of each image (28). This is a popular dataset for tasks involving pattern recognition in images which are also useful in health science. Even though this dataset is only available for free when used for research purposes, a similar scale of the dataset will be needed for applications that aim at addressing problems in healthcare for Africa. According to price listings by companies such as Scale.ai, annotating a single image will cost up to $\$ 6.40$ for semantic information. Hence, the cost associated with data acquisition is non-trivial (29).

2. Hardware and computing resources: AI is not a new technology. In fact, back-propagation, the algorithm upon which most powerful AI models are based was first introduced in $1975(30,31)$. The most recent breakthroughs in AI were only possible due to the convergence of powerful computing resources and the availability of massive data. That is, due to the introduction of special computer hardware such as dedicated graphics processing units (GPUs), it has become easier to process the high volume of calculations needed to 
train and run artificial neural networks faster than it has ever been done. Nowadays, the accuracy of a model can be predicted based on the magnitude of the available data and the computing power.

Unfortunately, this has also contributed to driving up the cost of developing new models and solutions, even creating new industry sectors such as Amazon Web Services and Microsoft Azure Cloud Computing Services that offer computing in the cloud for time-based fees. Depending on the computing resources needed for each project, the average cost could be up to $\$ 25$ per hour for a single GPU instance. Hence, a project can be expected to incur running costs ranging from $\$ 10,000$ to $\$ 30,000$ by the end of the year (32).

3. System maintenance and upgrading: This is the cost involved in ensuring that an AI model or application keeps working as expected when it is deployed. In practice, this is not always the case. The application, usually, will have to be changed in significant ways for it to keep up with the user expectations. The reason for this is largely due to the rate at which the technology itself changes. Since this is still a continuously growing technology, for research and industry, new tools are introduced more often, and old tools soon become outdated and need to be upgraded. According to the research by Sculley et al. (33), it was found that while machine learning offers a path to quickly engineering complex systems, the convenience comes with tremendous downstream costs.

\section{Inadequate Infrastructure}

Another major factor limiting the speed of development of this technology in Africa is inadequate infrastructure evident by low Internet penetration (39\%) and sociocultural factors impairing adoption (34). There is also widespread inaccessibility to electricity across the continent; about half of Africans have no access to electricity, and this has made it difficult to execute and sustain digital approaches in different sectors of the economy including healthcare (35). Adair-Rohani et al. (36) found out that less than $30 \%$ of health facilities on the continent had access to reliable electricity.

Digital health infrastructures-on which some of these MAI solutions may be built or integrated-have also been found to be inadequate (37). Time as an economic good is yet to be fully described in Africa's health space as the period spent designing a solution may hinge on whether it was rented, bought, or built. Some solutions are available off the shelf and require less time to deploy into healthcare, but complex health problems need to have custom-built AI solutions.

\section{Prospects}

A USAID report highlights four key areas where MAI might play an active role in Africa, namely, population health, individual care, health systems, and pharmaceuticals and medical technology (38). These encompass roles in improving the efficiency of programs ranging from immunization, logistics, and supply chain, referral services, diagnosis, drug safety and pharmacovigilance, and performance of limited medical procedures. With the worsening shortage of health workers, it is hoped that AI will help complement inadequate human resources through telehealth. Chatbots may minimize hospital visits and assist with triaging before medical consultation. Specially designed AI mobile applications requiring little skills can help in diagnosing birth asphyxia and malaria in the rural areas of Africa where there is a shortage of skilled health workers and medical equipment.

When electronic disease surveillance becomes more prevalent; MAI may be useful in the attenuation of epidemics, particularly in Africa where disease outbreaks are recurrent. The use of AI in administrative and clinical services can help to save time on routine tasks such as automation of adjunct activities like writing chart notes, prescribing medications, and ordering tests (39). Provision of personalized and efficient treatment through AIbased analysis of thousands of medical papers or publications will provide improved and up-to-date patient care and reduce operational costs.

\section{Recommendations}

We recommend the acceleration of the ongoing improvement in Africa's infrastructure especially electricity and Internet penetration. Reliable power supply and affordable Internet services will catalyze data generation and analysis needed for advanced automation of processes involved in patient care. Widespread use of electronic medical records and large medical databases will foster machine learning that is programmable for Africa.

By increasing the number of data scientists in Africa, a sizable workforce of experts will be made locally available to repurpose already existing AI for medical use. Citadels of learning should be adequately funded with more focus on bioinformatics, data science, computational biology, genomics, and applied mathematics. The field may become more attractive to prospective students if graduates of data science and related courses have better work opportunities in the marketplace. It is therefore imperative for private organizations to partner with educational institutions by providing internships before graduation.

With smartphone penetration projected to increase by over $100 \%$ in Africa over the next 30 years, AI developers need to put more focus on solutions that can be easily deployed on smartphones rather than on personal computers (40). Smartphones are cheaper, easier to power, and more portable for the myriads of health workers across the continent. Governments in African countries need to eschew disunity. By furthering cooperation between governments and states, trade, and commerce will grow, insecurity will be surmounted, and more resources will be available to create more robust, digitalized healthcare for the continent.

\section{CONCLUSION}

There is tremendous promise in the possibilities that AI offers in transforming and improving healthcare in low-resource areas like Africa. The existing use cases show that it is a viable tool for tackling health challenges, reducing costs, and improving health access and quality. Rather than mere enthusiasm to try out new methods, an evidence-based approach should be employed in decision-making and implementation of $\mathrm{AI}$ in healthcare. A 
major lesson from the experience of AI professionals working in resource-poor settings is that $\mathrm{AI}$ implementation should focus on building intelligence into existing systems and institutions rather than attempting to start from scratch or hoping to replace existing systems. African countries must also enact laws and policies that will guide the application of this technology to healthcare and protect the users.

\section{AUTHOR CONTRIBUTIONS}

AOw wrote the initial draft and worked on the literature search. JO contributed majorly to the writing of the technical

\section{REFERENCES}

1. Stuart R, Norvig P, Davis E. Artificial Intelligence: A Modern Approach. 3rd ed. Upper Saddle River, NJ: Prentice Hall (2010).

2. Samarghitean C, Vihinen M. Medical expert systems. Curr Bioinform. (2008) 3:56-65. doi: 10.2174/157489308783329869

3. Amisha, Malik P, Pathania M, Rathaur VK. Overview of artificial intelligence in medicine. J Fam Med Prim Care. (2019) 8:2328-31. doi: $10.4103 /$ jfmpc.jfmpc_440_19

4. Forbes Insights. AI and Healthcare: A Giant Opportunity. Available online at: https://www.forbes.com/sites/insights-intelai/2019/02/11/ai-andhealthcare-a-giant-opportunity/\#3afb11224c68 (cited November 24, 2019).

5. Hoodbhoy Z, Hasan B, Siddiqui K. Does artificial intelligence have any role in healthcare in low resource settings? J Med Artif Intell. (2019) 2:13. doi: $10.21037 /$ jmai.2019.06.01

6. Hunter J, Cookson J, Wyatt J, editors. AIME 89: Second European Conference on Artificial Intelligence in Medicine, London, August 29th-31st 1989. Proceedings. In: Lecture Notes in Medical Informatics, Vol. 38. Berlin, Heidelberg: Springer Berlin Heidelberg (1989). Available online at: http://link.springer.com/10.1007/978-3-642-93437-7 (cited April $25,2020)$.

7. Kastner JK, Dawson CR, Weiss SM, Kern KB, Kulikowski CA. An expert consultation system for frontline health workers in primary eye care. J Med Syst. (1984) 8:389-97. doi: 10.1007/BF02285251

8. Byass P. Computers in Africa: appropriate technology? Comput Bull. (1987) 3:17.

9. Forster D. Expert Systems in Health for Developing Countries: Practice, Problems, and Potential. Ottawa: International Development Research Centre (1992).

10. Moyo S, Doan TN, Yun JA, Tshuma N. Application of machine learning models in predicting length of stay among healthcare workers in underserved communities in South Africa. Hum Resour Health. (2018) 16:68. doi: 10.1186/s12960-018-0329-1

11. Brunskill E, Lesh N. Routing for Rural Health: Optimizing Community Health Worker Visit Schedules. In: 2010 AAAI Spring Symposium Series. (2010). Available online at: https://www.aaai.org/ocs/index.php/SSS/SSS10/ paper/view/1139 (cited November 24, 2019).

12. Onu CC, Lebensold J, Hamilton WL, Precup D. Neural transfer learning for cry-based diagnosis of perinatal asphyxia. Interspeech. (2019). doi: 10.21437/Interspeech.2019-2340

13. Bellemo V, Lim ZW, Lim G, P, Nguyen QD, Xie Y, Yip MYT, et al. Artificial intelligence using deep learning to screen for referable and vision-threatening diabetic retinopathy in Africa: a clinical validation study. Lancet Digit Health. (2019) 1:e35-44. doi: 10.1016/S2589-7500(19)30004-4

14. Melendez J, Philipsen RHHM, Chanda-Kapata P, Sunkutu V, Kapata N, van Ginneken B. Automatic versus human reading of chest X-rays in the Zambia National Tuberculosis Prevalence Survey. Int J Tuberc Lung Dis. (2017) 21:880-6. doi: 10.5588/ijtld.16.0851

15. Breuninger M, van Ginneken B, Philipsen RHHM, Mhimbira F, Hella JJ, Lwilla F, et al. Diagnostic accuracy of computer-aided detection of pulmonary challenges' aspect of the article. AOs contributed to writing all aspects of the article and edited the initial drafts. AB reviewed, edited, and made contributions on different aspects of the article. All authors contributed to the article and approved the submitted version.

\section{ACKNOWLEDGMENTS}

We will like to thank the Research Open Access Publishing (ROAAP) Fund of the University of Illinois at Chicago for the financial support towards the open access publishing fee for this article. tuberculosis in chest radiographs: a validation study from sub-Saharan Africa. PLoS One. (2014) 9:e106381. doi: 10.1371/journal.pone.0106381

16. Busari S, CNN and Segun Akande, for CNN. Nigerian Girls Win Silicon Valley Contest for App that Spots Fake Drugs. CNN. Available online at: https://www. cnn.com/2018/08/17/africa/nigerian-girls-win-silicon-valley-contest/index. html (cited November 24, 2019).

17. RxAll, Founded by Adebayo Alonge '16, Named Best Early-Stage Startup in Hello Tomorrow Global Challenge. Yale School of Management (2019). Available online at: https://som.yale.edu/news/2019/03/rxall-founded-byadebayo-alonge-16-named-best-early-stage-startup-in-hello-tomorrowglobal-challenge (cited November 24, 2019).

18. Tran BX, Vu GT, Ha GH, Vuong Q-H, Ho M-T, Vuong T-T, et al. Global evolution of research in artificial intelligence in health and medicine: a bibliometric study. J Clin Med. (2019) 8:360. doi: 10.3390/jcm8030360

19. Akanbi MO, Ocheke AN, Agaba PA, Daniyam CA, Agaba EI, Okeke EN, et al. Use of electronic health records in sub-saharan Africa: progress and challenges. J Med Trop. (2012) 14:1-6. Available online at: https://www.ajol. info/index.php/jmt/article/view/74802

20. Walsh CG, Chaudhry B, Dua P, Goodman KW, Kaplan B, Kavuluru R, et al. Stigma, biomarkers, and algorithmic bias: recommendations for precision behavioral health with artificial intelligence. JAMIA Open. (2020) 3:915. doi: 10.1093/jamiaopen/ooz054

21. Artificial Intelligence for Africa: An Opportunity for Growth, Development, and Democratisation. Pretoria: University of Pretoria, South Africa (2018). Available online at: https://www.up.ac.za/media/shared/7/ZP_Files/ai-forafrica.zp165664.pdf (cited November 24, 2019).

22. Mahomed S. Healthcare, artificial intelligence and the Fourth Industrial Revolution: ethical, social and legal considerations. South Afr J Bioeth Law. (2018) 11:93-5. doi: 10.7196/SAJBL.2018.v11i2.00664

23. Wahl B, Cossy-Gantner A, Germann S, Schwalbe NR. Artificial intelligence (AI) and global health: how can AI contribute to health in resource-poor settings? BMJ Glob Health. (2018) 3:e000798. doi: 10.1136/bmjgh-2018-000798

24. Every African Country's National eHealth Strategy or Digital Health Policy. ICTworks (2019). Available online at: https://www.ictworks.org/africannational-ehealth-strategy-policy/ (cited April 25, 2020).

25. Mitchell M, Kan L. Digital technology and the future of health systems. Health Syst Reform. (2019) 5:113-20. doi: 10.1080/23288604.2019.15 83040

26. Brandusescu A, Freuler J, Thakur D. Artifical Intelligence: Starting the policy dialogue in Africa. World Wide Web Foundation (2017). Available online at: http://webfoundation.org/docs/2017/12/Artificial-Intelligence-starting-thepolicy-dialogue-in-Africa.pdf (cited November 24, 2019).

27. How Much Does Artificial Intelligence (AI) Cost in 2019? AI, ML, NLP consulting and software development (2019). Available online at: https:// azati.ai/how-much-does-it-cost-to-utilize-machine-learning-artificialintelligence/ (cited November 24, 2019).

28. Lin T-Y, Maire M, Belongie S, Hays J, Perona P, Ramanan D, et al. Microsoft COCO: common objects in context. arXiv:1405.0312. (2014). doi: 10.1007/978-3-319-10602-1_48 
29. Pricing - Scale. Available online at: https://scale.com/pricing (cited January 17, 2020).

30. Courville A, Bengio Y, Goodfellow I. Deep Learning - Basics, Current Procedures and Algorithms, New Research Approaches. The MIT Press (2016). 801 p. Available online at: https://learning.oreilly.com/library/view/deeplearning-/9783958457027/Text/DRM.xhtml (cited January 17, 2020).

31. Werbos PJ. Beyond Regression: New Tools for Prediction and Analysis in the Behavioral Sciences. Washington, DC: Harvard University (1975).

32. Amazon EMR Pricing - Amazon Web Services. Amazon Web Services, Inc. Available online at: https://aws.amazon.com/emr/pricing/ (cited January 17, 2020).

33. Sculley D, Holt G, Golovin D, Davydov E, Phillips T, Ebner D, et al. Machine learning: the high interest credit card of technical debt. In: SE4ML: Software Engineering for Machine Learning (NIPS 2014 Workshop) Cambridge, MA (2014).

34. Halsey ED. What does AI actually cost? Medium. (2017). Available online at: https://medium.com/source-institute/what-does-ai-actually-costaf6a3e5a1795 (cited November 24, 2019).

35. More than Half of Sub-Saharan Africans Lack Access to Electricity. The Economist. Available online at: https://www.economist.com/graphicdetail/2019/11/13/more-than-half- of-sub-saharan-africans-lack-access-toelectricity (cited April 25, 2020).

36. Adair-Rohani H, Zukor K, Bonjour S, Wilburn S, Kuesel AC, Hebert R, et al. Limited electricity access in health facilities of sub-Saharan Africa: a systematic review of data on electricity access, sources, and reliability. Glob Health Sci Pract. (2013) 1:249-61. doi: 10.9745/GHSP-D-13-00037

37. Odekunle FF, Odekunle RO, Shankar S. Why sub-Saharan Africa lags in electronic health record adoption and possible strategies to increase its adoption in this region. Int J Health Sci. (2017) 11:59-64. doi: 10.12856/JHIA-2018-v5-i1-147

38. Artificial Intelligence in Global Health: Defining a Collective Path Forward. Washington, DC: USAID, The Rockerfella Foundation, Bill \& Melinda Gates Foundation (2019). Available online at: https://www.usaid.gov/sites/ default/files/documents/1864/AI-in-Global-Health_webFinal_508.pdf (cited November 24, 2019).

39. Zaidi D. The 3 Most Valuable Applications of AI in Health Care. VentureBeat. Available online at: https://venturebeat.com/2018/04/22/ the-3-most-valuable-applications-of-ai-in-health-care/ (cited January 17, 2020).

40. The Mobile Economy Sub-Saharan Africa. The Mobile Economy (2019). Available online at: https:/www.gsma.com/mobileeconomy/sub-saharanafrica/ (cited April 25, 2020).

Conflict of Interest: JO was employed by the company ZMP Inc., Japan.

The remaining authors declare that the research was conducted in the absence of any commercial or financial relationships that could be construed as a potential conflict of interest.

Copyright (c) 2020 Owoyemi, Owoyemi, Osiyemi and Boyd. This is an open-access article distributed under the terms of the Creative Commons Attribution License (CC $B Y)$. The use, distribution or reproduction in other forums is permitted, provided the original author(s) and the copyright owner(s) are credited and that the original publication in this journal is cited, in accordance with accepted academic practice. No use, distribution or reproduction is permitted which does not comply with these terms. 